TITLE:

\title{
Toward the stable optical trapping of a droplet with counter laser beams under microgravity
}

\section{$\operatorname{AUTHOR(S):~}$}

Isomura, Akihiro; Magome, Nobuyuki; Kohira, Masahiro I.; Yoshikawa, Kenichi

\section{CITATION:}

Isomura, Akihiro ...[et al]. Toward the stable optical trapping of a droplet with counter laser beams under microgravity. Chemical Physics Letters 2006, 429(1-3): 321-325

\section{ISSUE DATE:}

2006-09

URL:

http://hdl.handle.net/2433/49165

\section{RIGHT:}

Copyright (C) 2005 Elsevier B.V. All rights reserved.; この論文は出版社版 でありません。引用の際には出版社版をご確認ご利用ください。; This is not the published version. Please cite only the published version. 


\title{
Toward the Stable Optical Trapping of a Droplet with Counter Laser Beams under Microgravity
}

\author{
Akihiro Isomura, Nobuyuki Magome ${ }^{1}$, Masahiro I. Kohira ${ }^{2}$, \\ Kenichi Yoshikawa * \\ Department of Physics, Graduate School of Science, Kyoto University, Kyoto \\ 606-8502, Japan \& Spatio-Temporal Project, ICORP, JST, JAPAN
}

\begin{abstract}
To identify the optimum conditions for the optical trapping of a droplet under microgravity, we theoretically analyzed the efficiency of trapping with counter laser beams. We found that the distance between the two foci is an important parameter for obtaining stable trapping conditions. We also performed an optical trapping experiment with counter laser beams under microgravity. The experimental results correspond well to the theoretical prediction.
\end{abstract}

\section{Introduction}

Since the discovery of optical trapping in 1970 by Ashkin[1], optical tweezers have been actively applied in the fields of biology, physical chemistry, condensed matter physics and so on[2]. Up to the present era, increasing interests have been paid for the optical trapping in the air, as the useful tool under microgravity in the International Space Station (ISS)[3][4]. The technique of optical trapping is expected to be useful on the ISS for the manipulation of droplets on a $\mu \mathrm{m}$ to sub-mm scale, including in crystal growth by avoiding the effect of vessels. However, as far as we know, there has been no report on the optical trapping of a droplet under microgravity conditions in air.

\footnotetext{
* Corresponding author. FAX: +81 757533779

Email address: yoshikaw@scphys.kyoto-u.ac.jp (Kenichi Yoshikawa).

1 Department of Food and Nutrition, Nagoya Bunri College, Nagoya 451-0077, Japan.

2 Department of Physics, Chuo University, Kasuga, Bunkyo-ku, Tokyo 112-8551, Japan.
} 


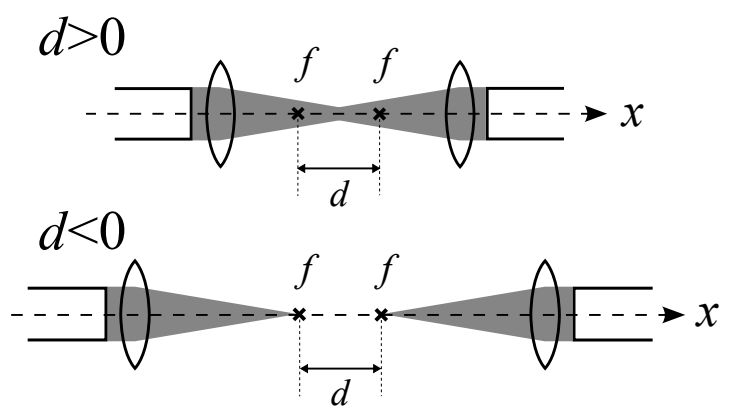

Fig. 1. Schematic illustration of the counter laser beams. $f$ is the focus of each laser beam and $d$ is the distance between them. We take the sign of $d$ as shown in the figure.

Recently, we reported the optical levitation of a droplet under a linear increase in gravitational acceleration using a single laser[5]. To attain stable optical trapping with a single beam, it is necessary to use a lens with a high magnification. This means that the working distance, or the distance between the lens surface and the object, is rather small; on the order of a few $\mathrm{mm}$. In view of such application on the ISS, it is important to find the stable trapping condition with a greater working distance. Toward this end, we adapted an optical system with low-converged counter laser beams for optical trapping with a long working distance.

\section{Theoretical}

We consider the counter laser system shown in Fig.1. In this system, the distance between the foci $d$ plays a crucial role. Although the qualitative property of such counter laser systems has been discussed in Ref.[6], the effect of the distance $d$ has not yet been discussed in detail. Here, we calculate the trapping force produced by counter lasers with the variables $d$ and $R$ (which is the radius of a spherical object).

To take into account the effect of the inertia of the object, we start our discussion with a motion equation. To simplify the problem, we consider motion along the $x$-direction as in Fig. 1 .

$$
m \ddot{x}=F_{l}+F_{v}+F_{\text {others }}
$$

where $m$ is the mass of the object, $\ddot{x}$ is the acceleration of the object along the $x$-direction, $F_{l}$ is the force along the optical axis induced by the converged laser, $F_{v}$ is the viscous force, and $F_{\text {others }}$ is the other forces (including the effective acceleration force along the $x$-direction and random force induced by air). 
As we see in the latter part, the velocity of the trapped object $U$ is at most $10^{-3} \mathrm{~m} / \mathrm{s}$. We assume that the scale of the trapped object $R$ is at most $10^{-4} \mathrm{~m}$, and the kinetic viscosity of air $\nu$ is around $10^{-5} \mathrm{~m}^{2} / \mathrm{s}$. In this condition, the Reynolds number is: $R e=R U / \nu \simeq 10^{-3} \ll 1$ and we can apply Stokes' approximation. By calculating $m=4 \pi R^{3} \rho / 3$ and $F_{v}=6 \pi \eta R v$, we can verify the relation $m v^{2} / 2 \ll F_{v} \cdot \Delta x$; where the radius of the trapped droplet $R$ is on the order of $10 \mu \mathrm{m}$, the experimental time resolution $\Delta t$ is $0.33 \mathrm{~s}$, the velocity of the trapped droplet $v=\Delta x / \Delta t$, the viscosity of air $\eta=1.8 \times 10^{-5} \mathrm{~Pa} \cdot \mathrm{s}$ and the density of the droplet $\rho=1.0 \times 10^{3} \mathrm{~kg} / \mathrm{m}^{3}$. This estimation indicates that the viscous force on our experimental condition is large enough to locate the droplet at an equilibrium position in the optical potential. Therefore, under reasonable assumptions of the viscous limit, we discuss the stability of trapping droplets through $F_{l}$ and the effective optical potential.

$F_{l}$ is found as follows. In the case of trapping a large object $(R / \lambda>10$, where $\lambda$ is the wavelength of laser), we can calculate the trapping force with the ray optics theory. Within the framework of ray optics, a TEM To $_{\text {mode laser can }}$ be divided into rays, which are suffixed with $i$, and each power of the ray $P_{i}$ is related to the beam deviation $\sigma$ and lens size $L$ Each ray hits the surface of the droplet at a different incident angle $\phi_{i}\left(0 \leq \phi_{i} \leq \phi\right)$, repeatedly reflects and is transmitted in the droplet until the intensity of the rays decreases to the zero limit, and the momentum is given with a certain efficiency along the $x$-axis, $Q_{i}=Q_{i}\left(R, x ; \phi_{i}, n_{1}, n_{2}\right)$ :

$$
\begin{array}{r}
Q_{i}=\sin \phi_{i}\left\{R_{i} \sin 2 \theta_{i}-\frac{T_{i}^{2}\left[\sin \left(2 \theta_{i}-2 r_{i}\right)+R_{i} \sin 2 \theta_{i}\right]}{1+R_{i}^{2}+2 R_{i} \cos 2 r_{i}}\right\} \\
+\cos \phi_{i}\left\{1+R_{i} \cos 2 \theta_{i}-\frac{T_{i}^{2}\left[\cos \left(2 \theta_{i}-2 r_{i}\right)+R_{i} \cos 2 \theta_{i}\right]}{1+R_{i}^{2}+2 R_{i} \cos 2 r_{i}}\right\}
\end{array}
$$

where $\theta_{i}$ : incident angle, $r_{i}$ : refractive angle, $R_{i}$ :reflection coefficient and $T_{i}$ : transmission coefficient. These parameters are obtained by considering the geometric relation between the droplet and the direction of the beam, expressed by $R, x, \phi_{i}, \frac{\sigma}{L}, n_{1}, n_{2}$ (see ref.[7]). Assuming a spherical droplet with radius $R$ whose center is located at distance $x$ from the center of the foci, we calculate the details of the reflections and transmissions for all paths of the laser beam. The total force $f_{l}$ is

$$
f_{l}=\sum_{i} \frac{n_{1} P_{i}}{\mathrm{c}} Q_{i}=\frac{n_{1} P}{\mathrm{c}} Q_{x}
$$

where $Q_{x}=Q_{x}\left(R, x ; \phi, \frac{\sigma}{L}, n_{1}, n_{2}\right)$ : trapping efficiency along the optical axis and $c$ : velocity of light. In addition, considering the distances between the 
(a)

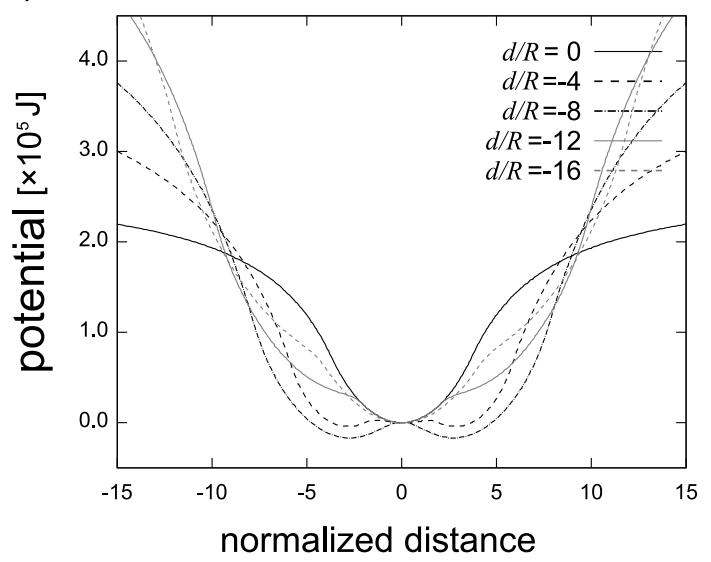

(b)

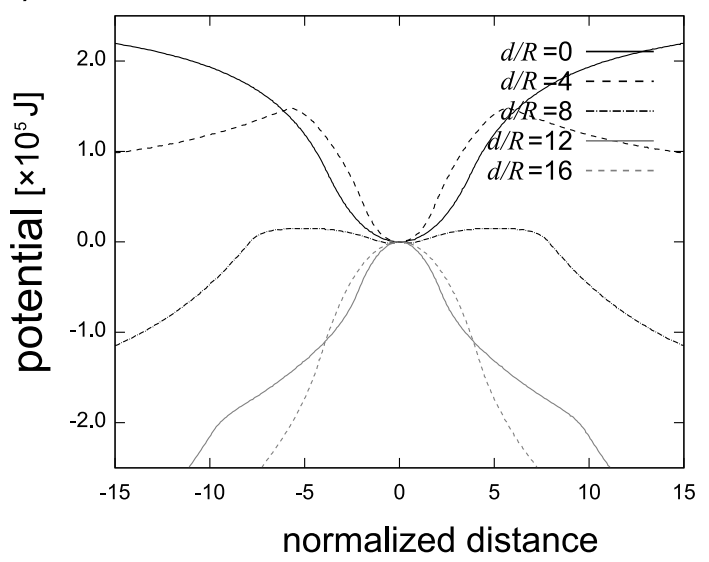

(c)

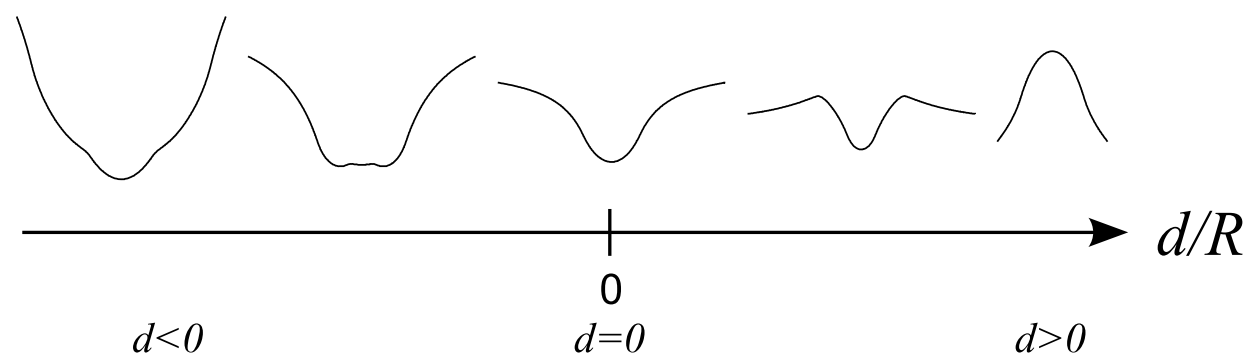

Fig. 2. Calculated potential of the counter beam lasers, deduced by a theoretical calculation. (a) $d<0$. (b) $d>0$. In the theoretical calculation, the following parameters were used: laser power $P=150 \mathrm{~mW}$, convergence angle $\phi=17.0$ degrees, injected ratio of laser to the lens $\sigma / L=1.5$, refractive index of the medium $n_{1}=1.00$, refractive index of the droplet $n_{2}=1.35$, density of the droplet $\rho=9.65 \times 10^{2} \mathrm{~kg} / \mathrm{m}^{3}$. (c)Schematic illustration of the calculated potential ((a) and (b)) made by the two-beam lasers, considering the radius of the droplet and the disagreement between the axes.

foci, the total force $F_{l}$ is:

$$
F_{l}(d, x, R)=f_{l}^{\text {Right }}+f_{l}^{\text {Left }}
$$

Under the assumption of a spherical trapped object, optical trapping exhibits geometrical symmetry and we can use a normalized unit of length. Eventually, the optical potential is obtained from the integration of $F_{l}$ and expressed by two normalized parameters, $d / R$ and $x / R$.

We calculated potential of the counter beam lasers, using the parameters: laser power $\mathrm{P}=150 \mathrm{~mW}$, convergence angle $\phi=17.0$ degrees, injected ratio of laser to the lens $\sigma / L=1.5$, refractive index of the medium $n_{1}=1.00$, refractive index of the droplet $n_{2}=1.35$, density of the droplet $\rho=9.65 \times 10^{2} \mathrm{~kg} / \mathrm{m}^{3}$. The results of the calculation for effective trapping potential are shown in Fig.2, indicating that the trapping efficiency is significantly dependent on $d$. An optical configuration with positive $d$ is clearly preferable for optilcal trapping 
(a)
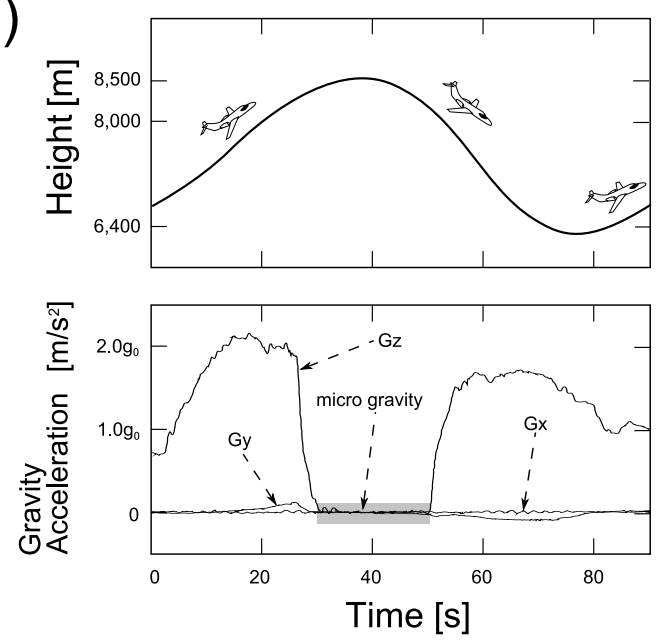

(b)

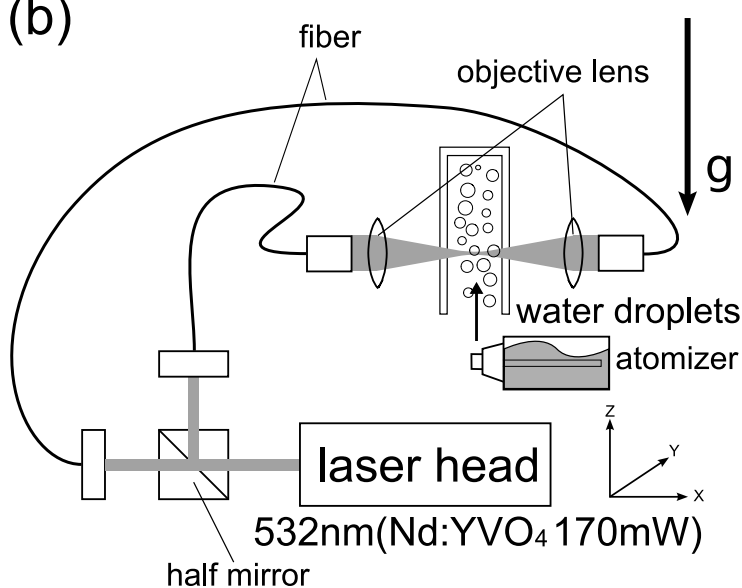

Fig. 3. (a) Schematic illustration of parabolic flight (top) and the actual change in gravity with the present parabolic flight profile(bottom). (b) Schematic illustration of the optical trapping system.

under microgravity. Figure 2(c) shows the change in the potential profile as a function of $d / R$. As shown, not only the distance between the foci but also the radius of the droplet greatly affect the profile of the optical potential. This chart is reasonable for understanding qualitative properties: if the radius $R$ is sufficiently large, the trapped object is insensitive to the distance between the foci $d$ and the sign of $d$ become less important. In contrast, when $R$ is sufficiently small, $d$ becomes non-negligible and we must choose better settings of $d$. Since we sought to define the optimum conditions for trapping large objects (sub-mm scale), this diagram is applicable for designing a counter laser trapping system. For example. if we can precisely adjust the positions of the foci, the setting at $d / R=4$ is advantageous for trapping the object rather than $d / R=-4$. The potentiality to trap small particles is important in the growth of protein crystals[8][9] and liquid droplets[10].

\section{Experimental}

Microgravity conditions were achieved by using a jet airplane(Mitsubishi MU300, operated by Diamond Air Service Co., Aichi, Japan). In the experiment, the airplane flew along a parabolic flight profile, as shown in Fig.3. During parabolic flight, the effective gravitational acceleration $g$ in the airplane was $0.01 g_{0}$ for about $20 \mathrm{~s}$, where the gravitational acceleration on the ground $g_{0}=9.8 \mathrm{~m} / \mathrm{s}^{2}$.

The experimental setup for the optical trapping is shown in Fig.3(b). We selected a counter laser system to press the droplets to the center of the foci.[11][12] The laser beam from a diode pumped $\mathrm{Nd}: \mathrm{YVO}_{4}(\lambda=532 \mathrm{~nm})$ 
was split into two beams by a half mirror, transferred via optical fibers, and focused by achromatic lenses. The convergence angle of the lenses was 17 degrees, so the working distance was on the order of $\mathrm{cm}$. The laser power was set to $170 \mathrm{~mW}$. We arranged two different optical settings, where the positions of the laser foci are different, either $d$ is positive or negative as shown in Fig.1. The droplets were injected into a glass cell $(10 \mathrm{~mm} \times 10 \mathrm{~mm} \times 50 \mathrm{~mm})$ with an atomizer. The droplets, as visualized by scattering visible laser light, were monitored with a CCD camera from the y-direction in Fig.3(b). Movies were recorded at 30 frames per second. The experiments was carried out at arround $298 \mathrm{~K}$.

\section{Results and Discussion}

On the ground in air, using the same optical trapping system, the injected droplets immediately fell due to the force of gravity. However, under microgravity in air, most of the droplets didn't fall and we could observe the motions of the droplets along the laser axes. To trace the droplets' motion along the laser axes, we converted the movie images to a spatio-temporal diagram. Figure 4 shows the results of the optical trapping of the droplets under microgravity.

Figure 4 (a1) and (b1) are spatio-temporal diagrams which show the trajectory of the droplets. Figure 4 (a2) and (b2) are snapshots of the droplets. In these figures, the burring on the images correspond to rather fast motion of the droplets. The small white spots are droplets that have departed from the laser axes and are floating in front of or behind the camera foci. In contrast, a trapped droplet is a star-like object(white arrow), since a trapped droplet on the laser axes scatter light intensively. In other words, the droplet was trapped in the setting at $d / R>0$ and was not in the setting at $d / R>0$.

Figure 4(c) shows typical profiles depicted based on the results of Fig.2(a) and (b), which are associated with the two different optical settings in Fig.4(a2) and (b2). The potential well in the case of $d / R=-4$ is deeper than that of $d / R=4$, as a whole. Whereas, near the trapping center, the gradient force in the case of the solid $\operatorname{arrow}(d / R=4)$ is much greater than that of the dashed $\operatorname{arrow}(d / R=-4)$. These results show that the optical setting $d / R>0$ has the advantage over the setting $d / R<0$ because of the narrow, deep potential profile near the trapping center. It is clear that such a theoretical expectation on on the advantage of the former setting corresponds well to the experimental results, given in Fig.4(a2) and (b2). 
(a1) $x$-direction

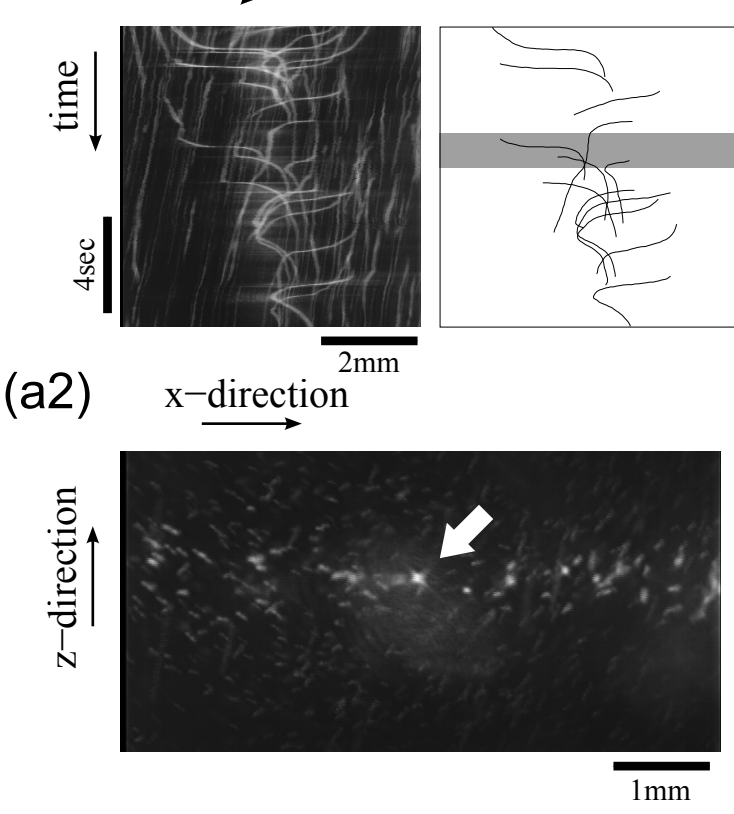

(b1)
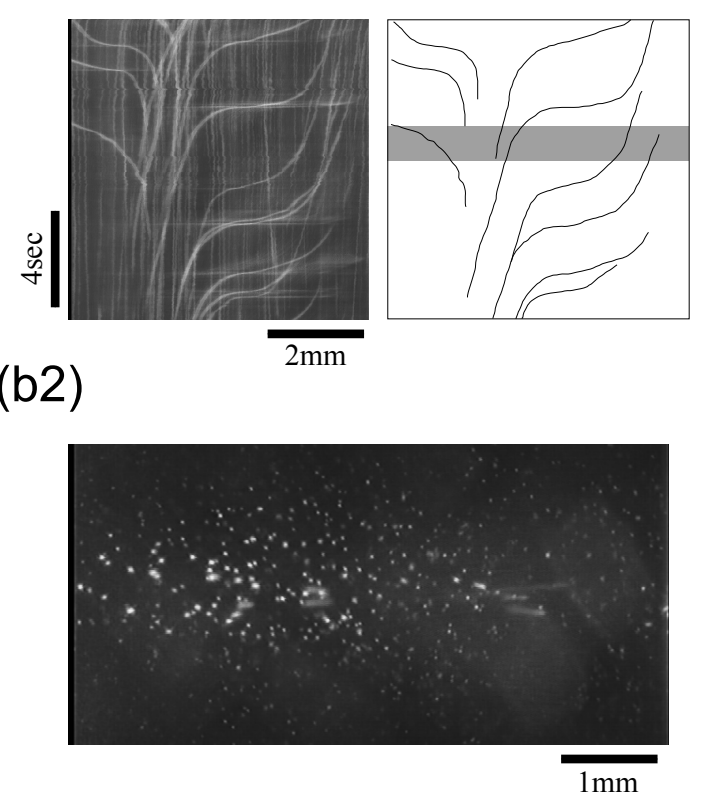

(c)

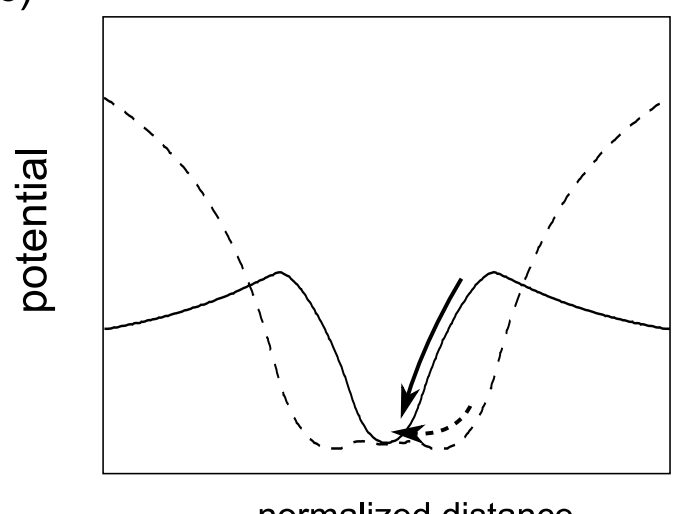

normalized distance

Fig. 4. Spatio-temporal diagrams of droplets under microgravity conditions together with selected representations of traces. The optics are (a1) $d>0$. (b1) $d<0$. (a2) and (b2) are the $1 \mathrm{sec}$ accumulation of the video frames, indicating the existence of a trapped particle in the former, whereas no particle was fixed in the latter. (c) Schematic illustration to explain the difference between trapped and untrapped droplets. The solid line is the potential profile in the case of $d / R=4$ and the broken line is that in the case of $d / R=-4$.

\section{Conclusion}

We performed the optical trapping of a water droplet with a counter laser in air under microgravity and investigated the properties of the counter laser trapping system. We showed that the position of the laser foci strongly affected the trapping efficiency. A theoretical calculation based on ray optics 
reproduced the experimental trend. The working distance of our trapping system is on the order of $\mathrm{cm}$, so it has the advantages of trapping large objects. The working distance of our trapping system was on the order of $\mathrm{cm}$, indicating the significant advantage compared to the single-laser trapping. The present results may contribute to the design of a manipulation system on the International Space Station (ISS), including experiments on protein crystal growth and a container-less micro-reactor.

\section{Acknowledgement}

The authors thank Mr. S. Watanabe, Mr. Y. Sumino, and N. Matsuda for their helpful suggestions and Ms. Hayata and Messrs. Fujii, Kawakatsu, and Takahashi for their technical assistance. This research was supported in part by a Grant-in Aid from the Ministry of Education, Science, Sports and Culture of Japan, the Grant-in-Aid for the 21st Century COE“ Center for Diversity and Universality in Physics" from the Ministry of Education, Culture, Sports, Science and Technology (MEXT) of Japan, and " Ground-based Research Announcement for Space Utilization” promoted by the Japan Space Forum.

\section{References}

[1] A. Ashkin, Acceleration and trapping of particles by radiation pressure, Phys. Rev. Lett. 24 (1970) 156.

[2] D. G. Grier, A revolution in optical manipulation, Nature 424 (2003) 810.

[3] J. Panda, C. R. Gomez, Setting up a rayleigh scattering based flow measuring system in a large nozzle testing facility, NASA/TM (2002) 211985.

[4] S. Y. Wrbanek, K. E. Weiland, Optical levitation of micro-scale particles in air, NASA/TM (2004) 212889.

[5] M. I. Kohira, A. Isomura, N. Magome, S. Mukai, K. Yoshikawa, Optical levitation of a droplet under a linear increase in gravitational acceleration, Chem. Phys. Lett. 414 (2005) 389.

[6] G. Roosen, A theoretical and experimental study of the stable equilibrium positions of spheres levitated by two horizontal laser beams, Opt. Comm. 21 (1977) 189.

[7] A. Ashkin, Forces of a single-beam gradient laser trap on a dielectric sphere in the ray optics regime, Biophys. J. 61 (1992) 569. 
[8] R. Giegé, J. Drenth, A. Ducruix, A. McPherson, W. Saenger, Study of the aerodynamic trap for containerless laser materials processing in microgravity, Prog. Cryst. Growth Charact. Mater. 30 (1995) 237.

[9] Y. Hosokawa, S. Matsumura, H. Masuhara, K. Ikeda, A. Shimo-oka, H. Mori, Laser trapping and patterning of protein microcrystals: Toward highly integrated protein microarrays, J. Appl. Phys. 96 (2004) 2945.

[10] N. Magome, M. I. Kohira, E. Hayata, S. Mukai, K. Yoshikawa, Optical trapping of a growing water droplet in air, J. Phys. Chem. B 107 (2003) 3988.

[11] G. Roosen, C. Imbert, Optical levitation by means of two horizontal laser beams: A theoretical and experimental study, Phys. Lett. 59A (1976) 6.

[12] T. Cizmar, V. Garcés-Chávez, K. Dholakia, P. Zemánek, Optical conveyor belt for ddelivery of submicron objects, Appl. Phys. Lett. 86 (2005) 17401. 